\title{
Population Biology and Criticality
}

From Critical Birth-Death Processes

to Self-Organized Criticality in

Mutation Pathogen Systems

○ 


\section{Population Biology and Criticality}

\section{From Critical Birth-Death Processes to Self-Organized Criticality in Mutation Pathogen Systems}

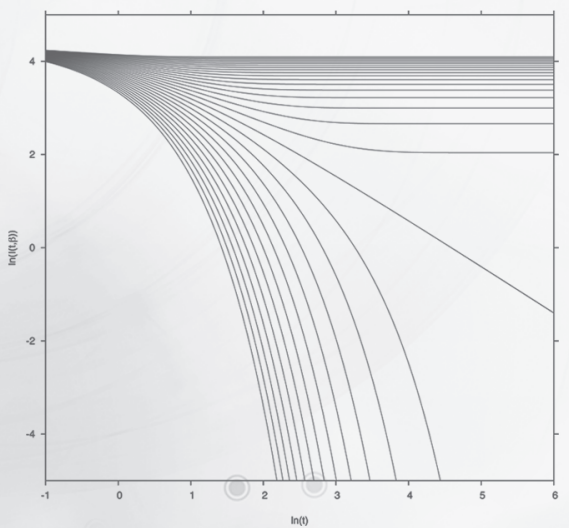

$\odot$

\section{Nico Stollenwerk}

Universidade de Lisboa, Portugal

\section{Vincent Jansen}

Royal Holloway, University of London 


\section{Published by}

Imperial College Press

57 Shelton Street

Covent Garden

London WC2H 9HE

\section{Distributed by}

World Scientific Publishing Co. Pte. Ltd.

5 Toh Tuck Link, Singapore 596224

USA office: 27 Warren Street, Suite 401-402, Hackensack, NJ 07601

UK office: 57 Shelton Street, Covent Garden, London WC2H 9HE

\section{British Library Cataloguing-in-Publication Data}

A catalogue record for this book is available from the British Library.

\section{POPULATION BIOLOGY AND CRITICALITY \\ From Critical Birth-Death Processes to Self-Organized Criticality in Mutation Pathogen Systems}

Copyright (C) 2011 by Imperial College Press

All rights reserved. This book, or parts thereof, may not be reproduced in any form or by any means, electronic or mechanical, including photocopying, recording or any information storage and retrieval system now known or to be invented, without written permission from the Publisher.

For photocopying of material in this volume, please pay a copying fee through the Copyright Clearance Center, Inc., 222 Rosewood Drive, Danvers, MA 01923, USA. In this case permission to photocopy is not required from the publisher.

ISBN-13 978-1-84816-401-7

ISBN-10 1-84816-401-7

Printed in Singapore. 


\section{Preface}

This book describes novel theories of mutation pathogen systems showing critical fluctuations as a paradigmatic example of an application of the mathematics of critical phenomena to the life sciences. It will enable the reader to understand the implications and future impact of these findings, and at same time allow them to actively follow the mathematical tools and scientific origins of critical phenomena. Hopefully this will lead to further fruitful applications of the mathematics of critical phenomena in other fields of the life sciences.

We show through explicit calculations the basis for understanding critical fluctuations as a genuine effect of stochastic dynamic systems, including the original background in statistical physics. Birth and death processes occur in many applications in the life sciences, in ecological and epidemiological systems and cancer growth, for example. In such simple systems the critical threshold appears and can be analyzed in all its different aspects.

Guided by our own research experience we then proceed to a system of pathogens evolving towards a critical state in a multi-mutant system. Although we concentrate on a special case study, the mechanism will certainly have much wider implications theoretically, understanding the mechanisms leading to systems with large fluctuations, as well as empirically, as in many real world systems such large fluctuations are observed but their mechanism often not recognized as such or not well understood.

To enable researchers in the various fields of application to analyze their systems appropriately we show explicitly the calculations and mathematical tools involved, which are now widespread in the textbook literature and the original scientific literature in journals. Since they are often spread over a wide range of scientific fields they use very different language and notations. 
From our own teaching experience we see the necessity of taking the reader from basic notions of stochastic dynamics, often taught to physics students and probability theoreticians but rarely to students from the life sciences, to a detailed analysis of the birth and death process, which is often taught to biologists and other life scientists, but rarely in the aspect of criticality. The field of critical phenomena in birth and death processes itself is a branch of rather high specialization in statistical physics with loose applications mainly in condensed matter physics or no application directly in mind.

However, when looking at real world data, large fluctuations are often observed, and cannot always be traced back to large external influences, hence the need to search for possible internal mechanisms. These internal mechanisms are given by the theory of critical phenomena, as we will explicitly demonstrate in our paradigmatic case study.

This book contains two parts. The first includes an introduction to stochastic dynamic systems and criticality, the second explores the application of mutation pathogen systems as a case study for wider applications.

We thank our students, José Martins, Maíra Aguiar, Rui Gonçalvez and Sander van Noort, for many discussions and calculations, and many collegues of ours for inspiration, especially but by no means exclusively Friedhelm Drepper, Minus van Baalen, Martin Maiden, Alberto Pinto, Gabriela Gomes, Frank Hilker, Lewi Stone, Walter Nadler and Luis Sanchez.

N. Stollenwerk and V. Jansen Lisbon and London, August 2009 


\section{Contents}

Preface $\quad$ v

1. From Deterministic to Stochastic Dynamics 1

1.1 Basic Probability Theory: The Tool Box . . . . . . . . . . . 1

1.2 Stochastic Description of a Deterministic System: The Ulam Map . . . . . . . . . . . . . . . . . . 3

1.3 A Fully Stochastic Dynamic System: The AR(1)-Process . . 6

1.4 From Perron-Frobenius to Master Equation . . . . . . . 8

1.5 A First Example of a Master Equation: The Linear Infection Model ..................... 9

1.5.1 Solving the first example of a master equation . . . 11

1.5.2 Solution of the linear infection model . . . . . . . 13

1.5.3 Mean value and its dynamics . . . . . . . . . . . 14

1.5.4 Mean dynamics . . . . . . . . . . . . . . . . 15

1.6 The Birth and Death Process, a Non-Linear Stochastic System . . . . . . . . . . . . . . . . 16

1.7 Solution of the Birth-Death ODE Shows Criticality . . . 18

1.7.1 Numerical integration shows power law at criticality 20

1.7.2 Temporal correlation length diverges at criticality . . 21

2. Spatial Stochastic Birth-Death Process or SIS-Epidemics 23

2.1 The Spatial Master Equation . . . . . . . . . . . . . . 23

2.1.1 A first inspection of the spatial birth-death process . 24

2.2 Clusters and their Dynamics . . . . . . . . . . 27

2.2.1 Time evolution of marginals and local expectations . 30

2.3 Moment Equations . . . . . . . . . . . . . 32 
2.3.1 Mean field behavior . . . . . . . . . . . . . . . 34

2.3.2 Pair approximation . . . . . . . . . . . . . . . . . 37

2.4 The SIS Dynamics under Pair Approximation . . . . . . . . 42

2.5 Conclusions and Further Reading . . . . . . . . . . . . . 44

3. Criticality in Equilibrium Systems 45

3.1 The Glauber Model: Stochastic Dynamics for the Ising Model . . . . . . . . . . . . . . . 45

3.1.1 A first glance at the dynamic Ising model . . . . . . 48

3.2 The Ising Model, a Paradigm for Equilibrium Phase Transitions . . . . . . . . . . . . . . . 49

3.2.1 Distribution of magnetization and Gibbs free

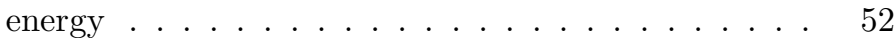

3.3 Equilibrium Distribution around Criticality . . . . . . 54

3.3.1 Distribution of magnetization . . . . . . . . . . 54

3.3.2 External magnetic field . . . . . . . . . . . . . . 55

3.3.3 The maximum of the total magnetization distribution $\quad 57$ 3.3.3.1 Approximation with Lagrange polynomials . 57

3.3.3.2 The maximum magnetization with changing parameters . . . . . . . . . 58

3.4 Mean Field Theory and its Exponents . . . . . . . . . . 60

3.4.1 Mean field self-consistency equation . . . . . . . . . . 61

3.4.2 Mean field quantities obtained from the selfconsistency equation . . . . . . . . . . . 64

3.4.2.1 Critical coupling strength in mean field approximation . . . . . . . . . . 64

3.4.2.2 The first critical exponent in mean field approximation . . . . . . . . 66

3.4.3 Universal scaling function and exponent $\delta$ in mean

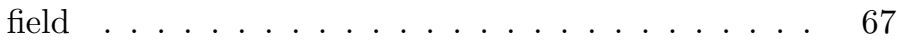

3.4.4 The magnetic susceptibility or second moment of the magnetization diverges . . . . . . . . . 68

3.4.5 State equation in mean field . . . . . . . . . . 70

3.5 Critical Exponents of the Ising Model beyond Mean Field . 72

4. Partial Immunization Models 75

4.1 A Model with Partial Immunization: SIRI . . . . . . . . . 76

4.2 Local Quantities . . . . . . . . . . . . . . . . 81 
4.3 Dynamics Equations for Global Pairs . . . . . . . . . . . 83

4.3.1 The SIRI dynamics under pair approximation . . . . 83

4.3.2 Balance equations for means and pairs . . . . . . . 84

4.4 Mean Field Model: SIRI with Reintroduced Susceptibles . . 86

4.4.1 Pair dynamics for the SIRI model . . . . . . . . . . 93

4.5 Fruitful Transfer between Equilibrium and Non-Equilibrium Systems . . . . . . . . . . . . . . . 93

5. Renormalization and Series Expansion: Techniques to Study Criticality $\quad 95$

5.1 Introduction . . . . . . . . . . . . . . . . . . . . 95

5.2 Real Space Renormalization in One-Dimensional Lattice Gas . . . . . . . . . . . . . . . . . . . 96

5.3 Directed Percolation and Path Integrals . . . . . . . . . 101

5.3.1 Master equation of the birth-death process . . . . . 102

5.3 .2 Schrödinger-like equation . . . . . . . . . . . . . . 103

$5.3 .3 \delta$-Bosons for hard-core particles . . . . . . . . . 105

5.3.4 Path integral for hard-core particles in a birth-death process . . . . . . . . . . . 106

5.4 Series Expansions . . . . . . . . . . . . . . . . . . . 107

5.4.1 The SIS epidemic model revisited . . . . . . . . . . 108

5.4.2 Perturbation analysis gives critical threshold . . . . . 109

5.5 Generalization to the SIRI Epidemic Model . . . . . . . . . 110

5.5.1 The SIRI epidemic model . . . . . . . . . . . . . . 113

5.5.2 Transitions in the SIRI model . . . . . . . . . . . . 114

6. Criticality in Measles under Vaccination 117

6.1 Measles around Criticality . . . . . . . . . . . . . . . . 117

6.2 The SIR Model . . . . . . . . . . . . . . . . . . . 118

6.2.1 The SIR model with vaccination . . . . . . . . . 120

6.2.2 Stationary states and vaccination threshold . . . . . 120

6.2.3 Definition and expression for the reproduction number $\mathcal{R} \ldots \ldots \ldots \ldots$. . . . . . . . . 122

6.2.4 Vaccination level at criticality $v_{c} \ldots \ldots \ldots \ldots$

6.2.5 Realistic parameters for measles epidemics . . . . . . 123

6.3 Stochastic Simulations . . . . . . . . . . . . . . . . 124

6.3.1 Stochastic bifurcation diagram for vaccine uptake . . 125

6.3.2 Large outbreaks during decreasing vaccine uptake . . 126 
7. Genetics and Criticality 129

7.1 Introduction . . . . . . . . . . . . . . . . . . . . . 129

7.2 Models in Genetics . . . . . . . . . . . . . . . . . . . . 131

7.2.1 The Moran model . . . . . . . . . . . . . . . . . . 132

7.2.2 The probability of fixation . . . . . . . . . . . 133

7.3 Mean Time until Fixation . . . . . . . . . . . . . . . 137

8. Evolution to Criticality in Meningococcal Disease 139

8.1 Accidental Pathogens . . . . . . . . . . . . . . . . . 140

8.2 Modeling Infection with Accidental Pathogens . . . . . . . 142

8.2.1 The meningitis model: SIRYX . . . . . . . . . . . 143

8.2.2 The invasion dynamics of mutant strains . . . . . . . 145

8.2.3 Divergent fluctuations for vanishing pathogenicity . . 147

8.3 Evolution toward Criticality . . . . . . . . . . . . . . . 149

8.3.1 Fast and slow time scales in the meningitis model . . 150

8.3.2 Analytics for evolution toward criticality . . . . . . . 151

8.3.3 Simulation for evolution toward criticality . . . . . . 153

8.3.4 Spatial modeling: Outbreak clusters without direct disease contacts . . . . . . . . . . . . 153

8.3.5 Mean pathogenicity goes toward zero . . . . . . . . 155

8.4 Empirical Data Show Fast Epidemic Response and Long-Lasting Fluctuations . . . . . . . . . . . . . . . . . . 158

8.4.1 Modeling fast epidemic response finds long-lasting fluctuations . . . . . . . . . . . . . 160

8.4.2 Data with fast epidemic response and long-lasting fluctuations . . . . . . . . . . . 166

Appendix A Invariant Density of the Ulam Map 169

A.1 Time Evolution Equation of the Density ....... . 169

A.2 Conjugation of Ulam Map and Tent Map . . . . . . . . . . 171

A.3 Exponential Divergence in the Ulam Map . . . . . . . . . . 173

Appendix B Parameter Estimation for the Autoregressive AR(1)-Process Gives Least Squares Estimators 175

B.1 Likelihood and its Maximization . . . . . . . . . . . . . . . 175

B.2 Bayesian Parameter Estimation . . . . . . . . . . . . . . . . 179

B.2.1 Conditional posteriors . . . . . . . . . . . . 180

B.2.2 Gibbs sampler . . . . . . . . . . . . . . 181 
Appendix C From Stochastic Epidemics to Parameter Estimation 183

C.1 Likelihood for Simple Stochastic Epidemic Model . . . . . 183

C.2 Calculation of the Likelihood Function . . . . . . . . . . . 185

C.3 Confidence Intervals via Inverse Fisher Matrix . . . . . . . 188

C.4 Improving Confidence Intervals . . . . . . . . . . . . . . . 191

Appendix D Product Space for Spin $1 / 2$

Many-Particle Systems

Appendix E Path Integral Using Coherent States

for Hard-Core Bosons

Appendix F Analytical Power Laws in the Meningitis Model 201

F.1 Distribution of Total Number of Cases . . . . . . . . . . . . 201

F.2 Scaling. . . . . . . . . . . . . . . . . . 203

F.3 Solution of Size Distribution of the Epidemic . . . . . . . . 205

F.4 Size Distribution of the Epidemic for $\varepsilon$ Zero . . . . . . . . 207

$\begin{array}{ll}\text { Bibliography } 213 & 213\end{array}$

$\begin{array}{ll}\text { Index } & 221\end{array}$ 